Voix et Images

volxetimages

PORTRAIT DE L'INTELLECTUEL « INTERMÉDIAIRE »

Engagement de la parole et du geste chez André Belleau

PORTRAIT OF THE “INTERMEDIARY” INTELLECTUAL

ANDRÉ BELLEAU'S COMMITMENT THROUGH WORDS AND

ACTIONS

RETRATO DEL INTELECTUAL 'INTERMEDIARIO'

COMPROMISO DE LA PALABRA Y DEL GESTO EN ANDRÉ

BELLEAU

\title{
JULIEN LEFORT-FAVREAU
}

Volume 42, numéro 2 (125), hiver 2017

André Belleau II : le texte multiple

URI : https://id.erudit.org/iderudit/1039912ar

DOI : https://doi.org/10.7202/1039912ar

Aller au sommaire du numéro

\section{Éditeur(s)}

Université du Québec à Montréal

\section{ISSN}

0318-9201 (imprimé)

1705-933X (numérique)

Découvrir la revue

Citer cet article

LEFORT-FAVREAU, J. (2017). PORTRAIT DE L'INTELLECTUEL

" INTERMÉDIAIRE » : engagement de la parole et du geste chez André Belleau.

Voix et Images, 42(2), 13-23. https://doi.org/10.7202/1039912ar

\section{Résumé de l'article}

Cet article vise à définir la notion d'intellectuel dans l'oeuvre théorique et critique d'André Belleau. Le terme " intermédiaire ", que nous utilisons pour circonscrire la notion, renvoie d'abord à la notion de code, telle que développée par Belleau dans « Code social et code littéraire dans le roman québécois ", où il met en relief le caractère intrinsèquement conflictuel du champ et de l'institution littéraires. Le terme « intermédiaire " renvoie également à la réflexion qu'entame Belleau sur les différents niveaux de culture. Belleau considère le rôle de l'intellectuel comme celui du " transcodeur ", c'est-à-dire celui qui sait interpréter la nouveauté politique des discours. En somme, Belleau met au point une théorie de l'intellectuel québécois qui aménage une zone de médiation entre l'engagement intellectuel et l'action militante, entre le geste et la parole. 


\section{PORTRAIT DE L'INTELLECT UEL \\ "I N T E R M É D I A I R E "}

Engagement de la parole et du geste

chez André Belleau

$+++$

JULIEN LEFORT-FAVREAU

Université de Sherbrooke/Université Queen's

Cet article vise à définir le concept d'intellectuel chez André Belleau, tâche quelque peu ardue en raison du caractère fuyant de la notion au fil de son œuvre. Le titre Y a-t-il un intellectuel dans la salle?, par son ironie peu assertive, met la puce à l'oreille. Belleau émet un doute: la fonction d'intellectuel est d'emblée définie par une absence potentielle, voire par une négation. Et s'il n'y avait aucun intellectuel dans la salle? On connaît l'anecdote restituée avec humour par Belleau : à l'automne 1981, Pierre Elliott Trudeau, s'adressant à des organisateurs politiques, leur lance, complice, la boutade suivante: «Il n'y a sûrement pas d'intellectuels dans la salle.» L'anti-intellectualisme de Trudeau pourrait s'avérer insignifiant s'il n'était pas évoqué dans un texte publié dans Liberté en 1983, que Belleau intitule justement: «Les écrivains québécois sont-ils des intellectuels?» Belleau y définit la notion d'intellectuel contre un discours social qui le marginaliserait ou qui le dépeindrait de manière péjorative. Belleau conclut ainsi ce court article, où le populisme d'Yves Beauchemin et de Claude Jasmin est l'objet de railleries: «Q: Les écrivains [québécois] sont-ils des intellectuels? R: Oui, mais l'ídéologie de leur société leur défend de l'avouer ${ }^{1}$.» Belleau, sans définir précisément son acception du terme, expose les rouages de son esprit fondamentalement dialectique: le point de référence pour identifier un intellectuel serait le discours qui l'empêche de l'être pleinement. Dans "La passion de l'essai», conférence prononcée en 1981 et publiée de manière posthume en 1987, il met en place cette curieuse ascèse: si l'écrivain est un intellectuel qui ne peut pas l'avouer, l'essayiste est pour sa part un intellectuel qui avoue l'être ${ }^{2}$. Pourquoi, en effet, s'arrêter en si bon chemin et s'entêter à séparer l'écrivain et l'intellectuel (reconduisant ainsi la distinction «quétaine» entre créateur et critique ${ }^{3}$ ), d'autant plus que les différences entre les deux fonctions ne semblent persister que par le biais d'une fantasmatique scène d'aveu?

Rachel Nadon remarque dans son essai La résistance en héritage une relative absence de Belleau dans les pages du Liberté contemporain, tout en notant une forme de descendance discrète:

1 André Belleau, «Les écrivains québécois sont-ils des intellectuels?» [1983], Surprendre les voix, Montréal, Boréal, coll. «Papiers collés», 1986, p. 157. Belleau souligne.

2 André Belleau, «La passion de l'essai», Liberté, vol. XXIX, n 1, 1987, p. 96.

3 André Belleau, «Petite essayistique» [1983], Surprendre les voix, p. 86. 
La position offensive et la filiation aquinienne que choisit le nouveau comité [entre 2006 et 2011] pour redéfinir Liberté laissent Belleau dans l'ombre; son legs se situe du côté d'une méthode de lecture, d'une éthique de la «recherche» et d'une pratique attentive des discours et des signes de la culture ${ }^{4}$.

Y aurait-il quelque chose au sein même de la définition de l'intellectuel qu'offre en pointillé Belleau qui empêcherait son appropriation? Y a-t-il quelque chose dans la conception de l'intellectuel élaborée par Belleau dès les années soixante et jusqu'à son décès qui rend difficile la remise en jeu de ses idées par une jeune génération? Je propose d'explorer quelques hypothèses à partir de la catégorie barbare d'«intellectuel intermédiaire» afin d'y voir plus clair.

\section{LE CONFLIT DES CODES}

Le terme «intermédiaire» renvoie d'abord à la notion de code, telle que développée par Belleau dans "Code social et code littéraire dans le roman québécois ${ }^{5}$ ", où il met en relief le caractère intrinsèquement conflictuel du champ et de l'institution littéraires. En effet, la première définition d'«intermédiaire» que je veux proposer correspond à l'idée que l'intellectuel est celui qui s'inscrit dans un champ polarisé, dans «le jeu réciproque des codes sociaux et des codes littéraires ${ }^{6}$ » d'une littérature québécoise doublement marginalisée par rapport à la France et à l'Amérique du Nord anglophone. L'institution québécoise est, à tout le moins en 1983, toujours incertaine dans son choix des codes - que ce soit dans le rapport qu'elle entretient avec la tradition (française) ou encore, plus généralement, avec une norme littéraire. Encore une fois, comme lorsqu'il évoque la question de l'intellectuel, Belleau offre une définition négative de l'institution. S'il décrit les «règles de production et de lisibilité des discours littéraires» en termes d'«empêchements», de "tensions», de "contradictions», de "non-dits», ce vocabulaire, précise-t-il, ne renvoie pas à une carence ou à une maladie, mais bien à une réponse «négociée ${ }^{7}$ » entre l'écrivain et sa société. Négociée serrée, a-t-on envie d'ajouter, tant l'espace littéraire a les apparences, sous sa plume, d'un véritable champ de bataille.

Cette structure trouve en effet de nombreux échos dans la posture d'essayiste que Belleau construit patiemment dans l'ensemble de son travail critique et théorique. De sa décision d'être «CONTRE [l]e CONTRE ${ }^{8}$ » des grandes entreprises en ce qui concerne l'indépendance du Québec, en passant par la figure de l'intellectuel contraint d'être «embarqué ${ }^{9}$ » dans une discussion sur l'autonomie de la Guadeloupe ${ }^{10}$

4 Rachel Nadon, La résistance en héritage. Le discours culturel des essayistes de Liberté (2006-2011), Montréal, Nota bene, coll. «Prémices», 2016, p. 185.

5 André Belleau, "Code social et code littéraire dans le roman québécois» [1983], Surprendre les voix, p. 175-192.

6 Ibid., p. 175.

7 Ibid., p. 177.

8 André Belleau, "Avant le référendum de 1980: l'esthétique du "oui" » [1980], Surprendre les voix, p. 99.

9 André Belleau, «Guadeloupe ambiguë» [1977], Surprendre les voix, p. 27. Belleau souligne.

10 Comment ne pas y voir une référence à l'écrivain engagé et «embarqué» sartrien? 
jusqu'à la célébration de la possibilité de se contredire comme vertu cardinale dans Liberté $^{11}$, il place toujours l'activité intellectuelle sous le signe d'une dialectique, d'une lutte entre adversaires. Cette dialectique le mène d'ailleurs naturellement vers une critique de l'anti-intellectualisme et de ses multiples avatars (chez les intellectuels eux-mêmes), bien plus que vers une définition normative de l'intellectualisme.

Cet agonisme constitutif a comme effet de mettre à distance toute forme d'essentialisation de la littérature. "[I]l n'y a de littérature que lorsqu'on en parle ${ }^{12}$ ", écritil dans le «Portrait du prof en jeune littératurologue». La fonction de l'intellectuel dans une telle économie agoniste de la littérature consisterait en premier lieu à en parler, et ce, dans les divers lieux où elle suscite un débat suffisant pour la constituer en objet conflictuel ${ }^{13}$. L'hétérogénéité du champ et de l'institution littéraires, indique très clairement Belleau, ne constitue pas une tare pour la littérature; c'est la condition même de son existence parce que ce sont dans ces conflits que l'écrivain négocie des solutions langagières aux problèmes provenant à la fois du monde social et des pressions de la double institution.

Le conflit des codes a certainement à voir avec une grille de lecture largement marxiste qu'André Belleau épouse de diverses manières. Relisant l'ensemble de ses essais, on peut observer une récurrence de la question des classes sociales, problème que Belleau ramène comme pour remettre les pendules à l'heure. Alors qu'il analyse les relations France-Québec, il déplace le point d'équilibre de son argumentation afin d'attirer l'attention sur le fait que l'«on a toujours négligé l'appartenance de classe dans la question des rapports entre les Français et les Québécois ${ }^{14} »$. Ailleurs, il évoque «l'abus de privilège» du professeur qui profite de sa position pour «mettre les rieurs de son côté ${ }^{15}$ ", remettant en cause l'attitude dominante de celui qui abuse de son autorité. Mais la sensibilité de Belleau à la question des classes sociales n'est pas un appel à la révolte des dominés sur les dominants. Son objet n'est pas l'économie et sa pensée ne met pas dos à dos les classes sociales pour proposer une solution de rechange au capitalisme. Le champ d'investigation de Belleau est culturel - ou, plus précisément, langagier.

Cette sensibilité aux diverses formes de domination réelles et symboliques ne peut, évidemment, faire l'économie d'une réflexion sur les différents niveaux de culture et leurs modes de diffusion spécifiques: «Ce que la société tolère mal, c'est qu'on dévoile et conteste le principe de [1']ordonnancement [des langages constitutifs du discours social ${ }^{16}$.» L'intellectuel se ferait justicier et se donnerait pour mission de révéler le fonctionnement des discours. Se dessine en creux une définition de l'intellectuel comme celui qui n'est pas inféodé à l'idéologie, en cela

11 André Belleau, «Liberté: la porte est ouverte» [1983], Surprendre les voix, p. 23.

12 André Belleau, «Portrait du prof en jeune littératurologue» [1980], Surprendre les voix, p. 91. L'auteur souligne.

13 Notons que, pour Belleau, l'intellectuel est essentiellement littéraire, ce qui apparaît comme l'un des traits caractéristiques de Liberté. Sa participation à la revue est déterminante pour lui et infléchit assurément sa conception de la vie intellectuelle.

14 André Belleau, «Parle(r)(z) de la France» [1981], Surprendre les voix, p. 34.

15 André Belleau, «Portrait du prof en jeune littératurologue», p. 95.

16 André Belleau, «Culture de masse et institution littéraire» [1978], Surprendre les voix, p. 149. 
tout à fait conforme à la ligne éditoriale de Liberté à l'époque. Lorsque j'affirme que le cadre de Belleau est d'inspiration marxiste, $c^{\prime}$ est en gardant en tête sa méfiance à l'égard d'un "marxisme à sécurité maximale ${ }^{17}$ ». Marxiste sans être marxiste, en somme.

Michel van Schendel, quelques années après la disparition de Belleau, signe un long hommage à son ami dans lequel il inscrit le conflit des codes dans ce qu'il désigne comme la sémiosis sociale. Selon Van Schendel, si «Belleau avait un profond respect pour l'œuvre de Marx, du moins pour ce qu'il en connaissait ${ }^{18}$ ", sa pensée critique n'a rien de manichéenne. Contre un marxisme mécaniste qui anéantirait l'autonomie des procédés artistiques, Belleau ne fait pas d'adéquation entre les antagonismes de classes et les problèmes sociaux qui trouvent des résolutions (partielles) dans le langage:

Le collègue n'est pas benêt, il est lucide. Il a raison de rappeler une évidence: l'autonomie des formes sociales de langage et de culture par rapport aux conflits de classes. Si elles ne s'en différenciaient pas, si elles s'y absorbaient, l'étude distinctive de la parole et du geste deviendrait superflue, et l'histoire même des conflits, notre histoire, avorterait au seuil d'un sens audible. Il reconnaît d'ailleurs la césure, la lésion des totalités de principe ${ }^{19}$.

Van Schendel, dans sa longue exploration de la pensée de Belleau, rappelle que le caractère conflictuel de la littérature concerne au premier chef la manière dont le langage est traversé par des conflits qui révèlent des points de vue antagonistes sur le monde. Alors qu'il revient sur l'œuvre de Bakhtine et sa saisie critique par l'auteur de Notre Rabelais, il écrit:

Tous les interlocuteurs, non plus, n'ont pas la même attitude interprétative, et c'est peu dire qu'elle n'est pas la même, elle est le plus souvent conflictuelle. Le prix du beurre ou de l'huile, etc., les uns en parlent de haut, ils ont les moyens. D'autres, même pauvres, possèdent le moyen cultivé d'en parler analytiquement, abstraitement, luxueusement; ils recèlent ou dispensent une sorte de richesse. D'autres, enfin, parlent au ras de leurs petits moyens; ils sont conduits à confondre dans le signe du prix les qualités ustensiles du beurre. Des solidarités virtuelles se dessinent entre interlocuteurs mis en situation sociale d'énonciation, mais elles signalent dès leur annonce un conflit latent avec d'autres solidarités, avec des solidarités opposées, avec aussi des solidarités intermédiaires déchirées entre l'opposition et le partage $^{20}$.

L'intervention de Van Schendel éclaire en effet la manière dont la reprise du dialogisme sous la plume de Belleau rend compte d'une sensibilité à la situation sociale

17 André Belleau, «Portrait du prof en jeune littératurologue», p. 95.

18 Michel van Schendel, "Cher André (Portrait intellectuel d'un chercheur)", Rebonds critiques, t. I: Questions de littérature, Montréal, L’Hexagone, coll. «Essais littéraires», 1992, p. 113.

19 Ibid., p. 170.

20 Ibid., p. 87. 
d'énonciation. Le "spécialiste de littérature ${ }^{21}$ » observe une attitude interprétative intermédiaire, déchirée entre sa position et sa conscience de classe, entre l'aveu de son privilège de langage et la reconnaissance de l'hétérogénéité de la socialité du texte; plus encore, Van Schendel dessine les traits d'un intellectuel dont la responsabilité consiste à ne pas supposer de la capacité d'énonciation selon la classe sociale.

\section{ENTRE LES NIVEAUX DE CULTURE}

Pour mieux saisir le rapport ambivalent de Belleau au marxisme, ouvrons une brève parenthèse afin d'explorer la réflexion sur la "haute» et la «basse» culture élaborée dans son œuvre. Nous serons ensuite plus à même de voir les implications du «conflit des codes» sur le rôle de l'intellectuel. À propos de la culture, on constate rapidement que Belleau n'a aucune intention de suivre un cursus de lectures marxistes organisé, convoquant tantôt l'industrie culturelle adornienne ${ }^{22}$, puis sermonnant les lecteurs de Bakhtine qui se serviraient de ses théories pour relancer le "vieux débat entre haute culture [et] culture de masse ${ }^{23}$ ». Dans son investigation du fonctionnement de la culture, il me semble par moments très proche de Raymond Williams ${ }^{24}$, qu'il n'a probablement pas lu, qui n'est très assurément pas une référence explicite, mais qui semble néanmoins éclairer la conception - déjà très riche - de la culture chez Belleau. L'article de Belleau "Culture de masse et institution littéraire 25 » a en effet tout à voir avec le travail de Williams, il est vrai, assez peu connu à l'époque au Québec. Dans la pensée de Williams, la culture est envisagée comme un ensemble de pratiques, et n'obéit pas à une reproduction mécanique, contrairement au marxisme structuraliste qui lui est contemporain en sol français. La culture y est stratifiée en sous-catégories: la culture résiduelle, c'est-à-dire les idées du passé qui perdurent dans le présent et qui sont le plus souvent en adéquation avec la culture dominante; la culture émergente qui se faufile dans les failles bien étroites de la culture hégémonique; les pratiques alternatives et oppositionnelles qui, pour leur part, se définissent en marge des pôles hégémoniques de la culture. Williams conspue un manichéisme qui supposerait que les formes «légitimes» n'appartiennent qu'à une élite ou alors que les résidus «populaires» ne seraient que le produit d'une masse homogène et influençable. Morale de l'histoire: il n'existe pas de rapports abstraits entre culture et société, postulat que Belleau pourrait tout à fait faire sien.

Conséquemment, Williams propose sa petite théorie du langage qui ne confine pas le sujet à une impuissance devant l'idéologie qui l'interpelle. Il est peut-être utile de rappeler que, pour Belleau, la langue «populaire» n’est pas le joual. Michel van

\footnotetext{
21 Ibid.

22 André Belleau, «L'Allemagne comme lointain et comme profondeur» [1982], Surprendre les voix, p. 39-48.

23 André Belleau, «Carnavalesque pas mort?» [1984], Surprendre les voix, p. 196-197.

24 Pour avoir un aperçu de la pensée de Raymond Williams, voir Culture et matérialisme, traduit de l'anglais par Nicolas Calvé et Étienne Dobenesque, Montréal, Lux éditeur, coll. «Humanités», 2009, 248 p.

25 André Belleau, «Culture de masse et institution littéraire» [1978], Surprendre les voix, p. 149-154.
} 
Schendel restitue une anecdote qui semble en effet révéler une forme de mélancolie chez Belleau à l'égard d'une culture populaire en déclin, uniformisée par l'hégémonie culturelle:

Nous rentrions à Montréal par la route respirable du nord, à quatre dans la voiture. Aux abords de Trois-Rivières, le chemin se perdait. André le demanda à une dame âgée qui allait aux provisions. La réponse était précise. Chantal Gamache, Bernard Andrès et moi l'avions bien entendue. La voie suivait un fil blanc et des points de croix. André retenait le langage de la réponse: «La belle façon de parler», disait-il en se renversant sur le siège avant dont le poids risquait de m'écraser les cuisses. Il pensait à l'accent trifluvien, au chant des phrases, à une intonation, à une syntaxe rigoureuse, il pensait à une parole populaire qui n'a pas fait rémission. Voilà le contraire du joual, soulignait-il avec appétit; le joual est plaqué sur la langue comme une grossièreté de la mauvaise conscience bourgeoise ${ }^{26}$.

L'anecdote de Van Schendel montre bien que, pour Belleau, il n'y pas d'adéquation nette entre la classe et l'usage du langage. Le "peuple» fantasmatique de Belleau a des coutumes, un savoir-faire langagier, un discours carnavalesque qui n'a rien à voir avec la folklorisation du joual. Cette négation de la culture trouve des échos jusque dans l'«effet Derome», qui exclut la mère de Belleau («[c]'est à ce point que ma mère, qui ignore l'anglais, manque chaque soir la moitié du Téléjournal ${ }^{27}$ ») et qui participe à une «politique de l'acculturation ${ }^{28}$ ».

\section{L'INTELLECTUEL COMME TRANSCODEUR}

Dans ce lieu conflictuel et stratifié qu'est la littérature, quel est donc le rôle de l'intellectuel? Pour trouver une définition plus précise, il faut passer par le texte «Indépendance du discours et discours de l'indépendance ${ }^{29}$ ». Dans ce texte, Belleau fait le portrait d'un intellectuel qui sait naviguer entre les niveaux de culture et qui comprend leur agencement, hors d'une lecture marxiste qui ferait fi de leur structuration complexe, mais aussi hors d'une lecture qui ferait abstraction de la question des classes sociales et qui pécherait par dépolitisation, ou à tout le moins par manque de sensibilité pour les cultures subalternes. Dans cet essai, le «je» a une valeur "transindividuelle», ce qui ne le réduit pas pour autant à l'état de porteparole. Le refus d'usurper la parole des autres est symptomatique de sa méfiance à l'égard de l'intellectuel révolutionnaire qui parle au nom des autres (que ce soit un groupe ou une génération), geste contraire à la liberté fondamentale de l'écrivain. Comme il l'écrit à propos d'Amos Oz, l'égalité se manifeste également par une

26 Michel van Schendel, «Cher André (Portrait intellectuel d'un chercheur)», p. 164

27 André Belleau, «L'effet Derome ou comment Radio-Canada colonise et aliène son public», Liberté, vol. XXII, $n^{\circ}$ 3, mai-juin 1980, p. 4.

28 Michel van Schendel, «Cher André (Portrait intellectuel d'un chercheur)», p. 119.

29 André Belleau, «Indépendance du discours et discours de l'indépendance» [1984], Surprendre les voix, p. $125-140$. 
solidarité langagière qui s'étend à tous les membres d'une communauté, y compris le lecteur ${ }^{30}$.

Belleau tente donc dans cet article écrit dans la foulée du premier référendum sur l'indépendance du Québec de définir le discours sur l'indépendance en identifiant son répondant, sa composante dialogique - tout référendum, nous dit-il, est par nature manichéen. Parmi tous les moments de division dans une société, c'est l'affaire Dreyfus qu'il donne en exemple, événement politique qui incarne la naissance de l'intellectuel moderne, symbole fort de la conflictualité politique et de l'intervention des clercs dans la cité. Pour identifier avec précision où naît ce discours dialogique sur l'indépendance, il remonte sans surprise jusqu'à la Révolution tranquille, à ce qui a «modifié profondément le système de discours au Québec, [...] élargi le domaine de l'opinable, du pensable, de l'argumentable ${ }^{31} »$ :

On suppose toujours que les changements ayant quelque portée concrète se signalent en premier lieu dans le discours proprement politique ou dans le discours économique. Mais les choses ne s'avèrent pas aussi simples. Il peut arriver que la nouveauté politique, même la rupture politique, d'abord, apparaissent, transcodés, dans d'autres discours tel par exemple le discours littéraire ${ }^{32}$.

Les discours, nous apprend Belleau, assez œcuménique pour le coup, circulent; les thèmes "migrent d'un discours à l'autre», et un "équilibre nouveau se forme ${ }^{33}$ ». Malgré son apparent relativisme, Belleau désigne bel et bien une fonction précise des intellectuels, assumée durant la Révolution tranquille par les collaborateurs de Parti pris et de Liberté, soit celle d'intégrer des impensés au discours social. Ils ont décloisonné le champ de ce qui était concevable, en élargissant les discours existants (et dominants) sans séparer (quoique de manière très différente) littérature et discours politique. Évidemment, l'autre pôle de ce dialogisme est incarné par Cité libre, qui, pour sa part, agit plutôt en «machine à assimiler l'inassimilable à des catégories connues, fussentelles infamantes ${ }^{34} »$. Sont ainsi mis face à face Gérard Pelletier et Pierre Vadeboncoeur, le second supérieur au premier dans sa capacité à «interpréter des signes au lieu de réagir à des signaux ${ }^{35}$ ». Vadeboncoeur, devant la génération de Parti pris, a une réaction digne d'un intellectuel en prêtant l'oreille à leurs propos: «La jeune génération est différente de la nôtre en ce qu'elle ne préjuge de l'échec d'aucune idée ${ }^{36}$.» Il n'est pas inutile de rappeler que Belleau, accueillant les partipristes dans Liberté en 1963, a fait preuve d'une semblable bienveillance, en affirmant alors que la «littérature est un combat $^{37}$ ", transformant la revue en lieu à la fois dissensuel et ouvert.

30 André Belleau, «Petite grammaire de la solidarité avec le peuple» [1972], Surprendre les voix, p. 79-84.

31 André Belleau, «Indépendance du discours et discours de l'indépendance», p. 129.

32 Ibid.

33 Ibid., p. 130.

34 Ibid., p. 132.

35 Ibid., p. 133.

36 Pierre Vadeboncoeur, "Salutations d'usage», Parti pris, vol. I, n 1, octobre 1963, p. 52. Cité dans André Belleau, «Indépendance du discours et discours de l'indépendance», p. 133.

37 André Belleau, «La littérature est un combat», Liberté, vol. V, n² 2, mars-avril 1963, p. 82. 
Il établit également une distinction politiquement tranchée entre le véritable intellectuel, celui qui fait advenir le changement social dans le discours, et le mauvais intellectuel, complice du pouvoir. La méfiance de Belleau envers l'embrigadement des clercs ne se tourne pas seulement vers ceux à gauche qui ferment les yeux sur les idéologies totalitaires, mais aussi vers ceux qui, par la force du langage, se rangent du côté de l'oppression:

\begin{abstract}
Ceux qui avaient en 1964 un langage que le milieu de Cité libre voulait maintenir à toute force hors du langage sont ceux-là mêmes qui prirent le chemin des cellules en 1970. Les mêmes personnes qui, quelques années plus tôt, croyaient entendre le bruit des bottes dans les premiers essais de ma génération, ajoutèrent et raturèrent, sur les listes ignobles, les noms de ceux d'entre nous que la police devait emmener. $C^{\prime}$ est ainsi que l'on passe de la dénonciation à la délation ${ }^{38}$.
\end{abstract}

L'indépendance du discours advient lorsque les intellectuels sont capables d'accueillir la nouveauté, ce qui était préalablement impensable: «Il faut que certains événements arrivent dans le langage avant d'arriver dans la réalité. [...] Tout ce qui chez nous tend à diversifier, complexifier, étendre et renouveler le champ des discours travaille, en fin de compte, pour l'indépendance ${ }^{39}$." L'intellectuel a une fonction politique importante qui obéit à une logique du transcodage. Les idées émergent d'abord dans la littérature, et il est de la responsabilité de l'intellectuel de modifier leur code afin qu'elles adviennent dans le réel. Plus encore, il est celui qui se place entre la littérature (ou plus largement le discours) et un récepteur, faisant preuve d'une attention au lectorat qui rend compte d'un champ culturel défini par des pratiques, c'est-à-dire suivant les modes de réappropriation par les membres d'une communauté. L'intellectuel doit comprendre les discours, voire les complexifier. Belleau lui donne un rôle d'intermédiaire, au sens d'entre-deux, entre l'écrivain et le lecteur, entre le discours social et son possible interprétant. S'il semble par moments répugner à accorder une fonction politique à l'intellectuel, ici, la place qu'il lui réserve n'est certes pas celle de l'intellectuel engagé dans un parti, asservi à une cause; la responsabilité politique de l'écrivain est intimement liée à sa capacité à manier le langage en toute souplesse. Elle concerne, en dernière instance, les rapports entre le langage et le réel, elle consiste à lier les mots aux choses. Cloîtrer le discours, c'est confiner le réel. Pelletier et Trudeau ont d'abord empêché l'indépendance en la rendant illégitime dans le discours.

\title{
INTELLECTUEL INTERMÉDIAIRE OU INTELLECTUEL INACHEVÉ?
}

Belleau semble avancer à tâtons lorsqu'il définit l'intellectuel en sol québécois. En effet, le modèle de l'intellectuel révolutionnaire n'a pas la même signification au Québec qu'en France pour des raisons contextuelles sur lesquelles il n'est pas

38 André Belleau, «Indépendance du discours et discours de l'indépendance», p. 134.

39 Ibid., p. 139. 
nécessaire d'insister ${ }^{40}$. On a pu voir que, de manière générale, l'adhésion à une ligne de parti semble contraire à la polysémie qui domine son imaginaire politique. Il ne rejette pas le modèle de l'intellectuel critique, mais il serait abusif d'affirmer que Belleau se donne comme projet de remettre en question les fondements épistémologiques de sa discipline. Certes, il propose un certain nombre d'interventions théoriques des plus pertinentes, mais rien qui ne ferait de lui l'équivalent de ses contemporains français - Pierre Bourdieu, Jacques Derrida ou Michel Foucault. L'intellectuel de gouvernement n'a que peu de chances de trouver grâce aux yeux moqueurs de Belleau. Quant à l'intellectuel spécialiste, Belleau ne correspond pas à ce modèle: sa grande curiosité se manifeste notamment par un refus clair et net de s'enfermer dans un savoir spécifique qui le réduirait à l'état de demandeur de subventions numériques ${ }^{41}$.

Ces modèles français ne sont pas appropriés pour l'intellectuel québécois, en partie parce que si le champ intellectuel français est bien sûr polarisé (il suffit de lire La guerre des écrivains ${ }^{42}$ de Gisèle Sapiro pour comprendre que cette polarisation du champ ne se réduit pas à une joute stratégique et peut avoir des conséquences funestes), il n'est pas pour autant structuré par le conflit des codes à la québécoise et par sa double institution. L'insécurité linguistique et culturelle des Québécois exige, en effet, une alternative à l'intellectuel prophète guidant les foules vers l'émancipation. Elle impose un modèle plus souple, un modèle intermédiaire, où l'intellectuel est celui qui clarifie, qui ne camoufle pas la vérité, qui négocie des solutions langagières entre l'espace public et l'espace littéraire, qui reste conscient des problèmes de classes qui traversent la culture et le discours social. Le vrai intellectuel sait percevoir les nouvelles idées, la variété des possibilités du langage, il ne rejette pas la norme parce qu'il sait être narquois devant elle; il ne se construit pas véritablement contre la norme, il se construit dans la norme - avec beaucoup de «jeu» (au sens d'espace) dans la langue. Il est également intermédiaire d'un point de vue institutionnel, car il peut s'inscrire à la fois dans l'université (sans être spécialiste) et dans des lieux culturels (Radio-Canada, l'Office national du film, Liberté).

Que faire aujourd'hui avec une telle posture? L'intellectuel intermédiaire tel que j'ai tenté de le définir est-il encore possible en 2017? D'une part, on peut constater que le champ intellectuel actuel ne favorise par ces allers-retours entre la sphère académique et la diffusion auprès d'un public (au mieux) moins restreint. Déjà, en 1981, Belleau affirme: «[I]l est sûr que je pratique un type de discours que l'institution littéraire québécoise ne valorise pas autant que d'autres. Il m'arrive d'avoir le sentiment que j'aurais produit davantage si j'avais été dans un autre milieu ${ }^{43}$.» Belleau peut certes blâmer son milieu. Reste que sa postérité relativement discrète s'explique peut-être par une forme de poétique de l'inachèvement qui ne favorise malheureusement pas la descendance intellectuelle. François Dumont, dans un article

40 Je reprends ici la typologie établie par Gérard Noiriel, Dire la vérité au pouvoir. Les intellectuels en question, Marseille, Agone, coll. «Éléments», 2010 [2005], 316 p.

41 Du titre d'un article de Belleau, «Pourquoi je ne demanderai pas de subventions numériques pour des recherches digitales (et vice versa)» [1985], Surprendre les voix, p. 211-217.

42 Gisèle Sapiro, La guerre des écrivains, 1940-1953, Paris, Fayard, 1999, 807 p.

43 André Belleau, «La passion de l'essai», p. 95. 
de 1992 où il propose avec beaucoup de finesse une analyse de la collection «Papiers collés", remarque que

ce «culte du paradoxe» s'expliquerait sans doute par la méfiance que les contemporains entretiennent envers les systèmes clos et les utopies unitaires, lesquels n'auraient produit que déceptions. Mais la caractéristique de l'essai québécois contemporain serait peut-être moins la déception ou la destruction des certitudes que le fait de considérer l'inachèvement lui-même comme une fin, comme une garantie que le point d'arrivée est aussi un point de départ. Ainsi, faire de l'«essai littéraire», cela servirait moins à conclure qu'à continuer ${ }^{44}$.

Ce refus de "conclure» correspond tout à fait à la logique du transcodage évoquée plus haut. L'intellectuel du conflit des codes se doit de laisser les choses inachevées, afin de laisser ouvertes les interprétations. Cette logique s'apparente parfois, dans sa version douloureuse, au parcours du transfuge de classe, toujours mal à l'aise avec les codes alors que son versant utopique rend possible l'adoption d'identités politiques et intellectuelles, et favorise la résistance à tout essentialisme. Notons que le rapport de Belleau au nationalisme québécois semble teinté par ce refus de conclure.

En 1961, Belleau amorce déjà la réflexion qu'il poursuivra une vingtaine d'années plus tard:

Se demander s'il est désormais possible aux écrivains et intellectuels canadiensfrançais, en tant que groupe, - et à quelles conditions, - de traduire leurs idées et aspirations en une action susceptible de hâter la transformation de notre société, c'est poser du même coup le problème de l'efficacité... C'est émerger de nos représentations trop souvent globales et théoriques pour nous abandonner un instant à l'impatience dynamique et vitale de faire quelque chose tout de suite, sorte d'exigence absolue et douloureuse vis-à-vis des situations actuelles et concrètes dans un Canada français également actuel et concret, celui de $1961^{45}$.

Dans ce texte étonnant, dénué d'ironie, Belleau plaide pour l'action d'un «groupe» d'intellectuels, actif dans la réforme des institutions canadiennesfrançaises (notamment la création d'un ministère de l'Instruction publique), sensible aux inégalités sociales, investi dans le progrès de la culture. Ce qui frappe toutefois dans ce court article, c'est la tendance autocritique de Belleau, qui refuse de singulariser à outrance l'importance des intellectuels :

Après tout, ce n'est pas la masse, j'imagine, qui a inventé les mots "écrivains» et «intellectuels», et qui les arbore comme des étendards - et dans la mesure où ce que je viens de dire serait vrai, il y aurait peut-être une certaine intuition maladroitement exprimée dans les termes «pseudo» ou «soi-disant» intellectuels utilisés

44 François Dumont, "L'essai littéraire québécois des années quatre-vingt: la collection "Papiers collés" », Recherches sociographiques, vol. XXXIII, n² 2, 1992, p. 334.

45 André Belleau, «Action et enracinement», Liberté, vol. III, n 5, novembre 1961, p. 691. 
fréquemment chez nous. Si nos éternelles revendications ne se traduisent pas plus qu'elles ne le font en gestes positifs et palpables, aisément perceptibles au milieu dans son ensemble, il n'y aura plus précisément que les "pseudo » ou les «soi-disant » à pouvoir se faire appeler intellectuels ${ }^{46}$.

Les intellectuels doivent être utiles, voire servir une cause (celle de la Révolution tranquille et de la laïcisation de la société), à défaut de quoi ils ne deviendront que des simulacres, pâles copies des véritables penseurs. Mais ils ne doivent pas être asservis à des idéaux abstraits. La situation est urgente, et c'est la précarité du Canada français qui exige d'eux un tel engagement. C'est donc aussi dire que la société canadienne-française nécessite un modèle d'intellectuel bien à elle qui corresponde à sa réalité socioéconomique, à sa situation historique, à la place singulière des intellectuels dans celle-ci.

Agir de façon à atteindre un objectif précis parce que des problèmes très graves l'exigent dans les circonstances données de temps et de lieu, c'est donc soulever le problème de l'efficacité. C'est s'interroger sur les moyens, prévoir leurs effets dans l'immédiat, tenir compte des caractéristiques du milieu vivant ${ }^{47}$.

$L^{\prime}$ «action positive et efficace de la part des intellectuels ${ }^{48}$ » doit toutefois être "enracinée» (mot que Belleau emprunte à Maurice Blain) dans une connaissance historique. La notion d'intellectuel intermédiaire telle que je l'ai proposée ici mérite une dernière reformulation. L'intellectuel intermédiaire est celui qui est sans cesse pris entre l'action et la parole, conscient des limites de son action et de sa parole. Il cherche à définir la zone entre l'engagement sincère et la distance ironique, entre le désir d'utilité de sa parole et l'aveu de la limite de l'action restreinte, entre le lyrisme de l'engagement public et la discrétion de l'investissement institutionnel. L'intellectuel intermédiaire croit peut-être simplement, pour reprendre les mots d'Aquin que «la parole est une forme de vie et, par ce biais magnifique, un mode d'action ${ }^{49}$ ».

\footnotetext{
46 Ibid., p. 692. Belleau souligne.

47 Ibid.

48 Ibid., p. 695 .

49 Hubert Aquin, «Comprendre dangereusement», Liberté, vol. III, n 5, novembre 1961, p. 680. Notons que cet article est publié dans le même numéro de Liberté qu' «Action et enracinement».
} 